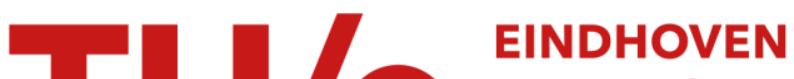 \\ UNIVERSITY OF \\ TECHNOLOGY
}

\section{Lattice heat capacity of low-dimensional systems}

\section{Citation for published version (APA):}

Kopinga, K., Van Der Leeden, P., \& De Jonge, W. J. M. (1976). Lattice heat capacity of low-dimensional systems: A pseudoelastic approximation. Physical Review B, 14(4), 1519-1530.

https://doi.org/10.1103/PhysRevB.14.1519

DOI:

10.1103/PhysRevB.14.1519

Document status and date:

Published: 01/01/1976

\section{Document Version:}

Publisher's PDF, also known as Version of Record (includes final page, issue and volume numbers)

\section{Please check the document version of this publication:}

- A submitted manuscript is the version of the article upon submission and before peer-review. There can be important differences between the submitted version and the official published version of record. People interested in the research are advised to contact the author for the final version of the publication, or visit the $\mathrm{DOI}$ to the publisher's website.

- The final author version and the galley proof are versions of the publication after peer review.

- The final published version features the final layout of the paper including the volume, issue and page numbers.

Link to publication

\section{General rights}

Copyright and moral rights for the publications made accessible in the public portal are retained by the authors and/or other copyright owners and it is a condition of accessing publications that users recognise and abide by the legal requirements associated with these rights.

- Users may download and print one copy of any publication from the public portal for the purpose of private study or research.

- You may not further distribute the material or use it for any profit-making activity or commercial gain

- You may freely distribute the URL identifying the publication in the public portal.

If the publication is distributed under the terms of Article 25fa of the Dutch Copyright Act, indicated by the "Taverne" license above, please follow below link for the End User Agreement:

www.tue.nl/taverne

Take down policy

If you believe that this document breaches copyright please contact us at:

openaccess@tue.nl

providing details and we will investigate your claim. 


\title{
Lattice heat capacity of low-dimensional systems: A pseudoelastic approximation
}

\author{
K. Kopinga, P. van der Leeden, and W. J. M. de Jonge \\ Department of Physics, Eindhoven University of Technology, Eindhoven, The Netherlands
}

(Received 7 July 1975)

\begin{abstract}
The lattice heat capacity of both layered and chainlike compounds has been inferred from an approximation of the vibrational spectrum, which is based upon a pseudoelastic approach. From continuum elasticity theory three modes of vibration are obtained, which are modified by the inclusion of the most dominant dispersion effects. Manageable expressions for the heat capacity are obtained, which were found to be of rather general applicability. They were used to describe the heat capacity of the chainlike diamagnetic $\left(\mathrm{CH}_{3}\right)_{4} \mathrm{NCdCl}_{3}$, and were found to give an excellent description of the lattice contribution to the heat capacity of a variety of low-dimensional magnetic substances.
\end{abstract}

\section{INTRODUCTION}

In the past years, a considerable number of compounds whose thermal behavior could be fairly well described by one- or two-dimensional model systems have been studied extensively. $.^{1-4} \mathrm{Es}-$ pecially in the field of magnetism, low-dimensional characteristics have received much attention.

The analysis of the thermal properties of a substance generally requires a separation of the lattice heat capacity from the other contributions. Although the lattice specific heat of low-dimensional systems with a simple crystallographic structure may be calculated rather straightforwardly, the majority of the low-dimensional compounds have rather complex chemical structures, which precludes a rigorous calculation of the frequency distribution of the lattice vibrations.

Fortunately, the lattice heat capacity appears to be rather insensitive to the detailed structure of the vibrational spectrum, and approximate spectrum calculations may provide a very satisfactory description in many cases. This is demonstrated by the fact that the overall lattice heat capacity of a large number of rather isotropic compounds can be successfully described by a linear superposition of suitably normalized threedimensional Debye functions. ${ }^{5}$

General and simple expressions for the lattice heat capacity of layered and chainlike structures have been proposed by Tarasov. ${ }^{6}$ Although his theory, in which the heat capacity is expressed as a linear combination of Debye functions of suitable dimensionality, contains a number of rather drastic simplifications, it correctly predicts some qualitative features of the overall heat capacity. However, in general the accuracy is not sufficient to enable a reliable separation of the magnetic and the lattice contribution to the heat capacity. ${ }^{7,8}$

In several cases the experimental data within a limited temperature region can be represented by a linear superposition of suitably normalized one-, two-, and three-dimensional Debye functions. In this kind of procedure, however, the Debye functions are merely used as mathematical basis functions, the normalization factors and $\Theta$ values being inferred from a least-squares fit to the experimental data. Apart from the fact that such a procedure lacks a physical background, an accurate description over a large temperature interval requires a rather large number of adjustable parameters. On the other hand, the experimental data on several pseudo-low-dimensional systems ${ }^{8-10}$ indicate that at lower temperatures the lattice heat capacity should be represented by higherorder terms than just $T^{3}$. This behavior cannot be described by a linear superposition of Debye functions, unless one admits rather unphysical values of the parameters.

Detailed calculations on the vibrational spectrum and thermal properties of strongly anisotropic compounds have been performed only in a few special cases, mostly dealing with layered structures, particularly graphite. ${ }^{11}$ Most of the results, however, cannot be applied to other substances, since they strongly depend on the characteristic lattice structure and the ratio of the atomic force constants. The purpose of this paper is to present a rather general description of the lattice heat capacity of both layered and chainlike compounds, involving only a minimum of adjustable parameters. The theory will be based upon an elastic approach, in which only the most dominant dis persion effects will be taken into account.

For a large variety of layered or chainlike compounds, the elastic anisotropy within the layers or perpendicular to the chains appears to be small compared to the anisotropy in a plane perpendicular to the layers or parallel to the chains. A fair integral description of the long-wavelength behavior of such compounds may therefore be pos- 
sible if they are approximated by a system with purely uniaxial elastic anisotropy, such as a hexagonal $6 / \mathrm{mmm}$ structure.

The organization of the paper is as follows. The dynamical behavior of media with uniaxial elastic anisotropy will be considered in Sec. II, while in Sec. III the frequency distribution function and the heat capacity of layered structures will be calculated. Section IV will be devoted to the heat capacity of chainlike structures. In Sec. $V$ the inferred expressions for the heat capacity will be confronted with some experimental results.

\section{LATTICE DYNAMICS IN UNIAXIAL COMPOUNDS}

The rather unusual temperature dependence of the specific heat of graphite was explained by Komatsu $^{12,13}$ by considering it as a system of loosely coupled layers. His basic idea was that, since the covalent binding forces within the honeycomb net planes are very strong compared to the interlayer interactions, dispersion effects in a direction perpendicular to the layers might already be important for a wide range of frequencies, in which waves propagating within the layers still could be treated in the elastic or small- $k$ approximation. In the calculation of the heat capacity, dispersion effects due to the discrete nature of the layers would therefore be negligible, and the substance might be treated as a system consisting of thin elastic plates spaced at a distance $d$. He described the restoring forces due to the intralayer interactions by the elastic constants $c_{11}, c_{12}$, and $c_{66}\left[=\frac{1}{2}\left(c_{11}-c_{12}\right)\right]$, and apart from these a bending modulus $K$. The restoring forces due to the interaction between the layers were represented by a compressional constant $c_{33}$ and a shearing constant $c_{44}$. For relatively small values of $c_{44}$, the following dispersion relations were inferred:

$$
\begin{aligned}
& \rho \omega_{1}^{2}=c_{11}\left(k_{x}^{2}+k_{y}^{2}\right)+\left(c_{44} / d^{2}\right) \sin ^{2}\left(k_{z} d\right), \\
& \rho \omega_{2}^{2}=c_{66}\left(k_{x}^{2}+k_{y}^{2}\right)+\left(c_{44} / d^{2}\right) \sin ^{2}\left(k_{z} d\right), \\
& \rho \omega_{3}^{2}=c_{44}\left(k_{x}^{2}+k_{y}^{2}\right)+\left(c_{33} / d^{2}\right) \sin ^{2}\left(k_{z} d\right)+K^{2}\left(k_{x}^{2}+k_{y}^{2}\right)^{2},
\end{aligned}
$$

where $z$ denotes the direction perpendicular to the layers, and $\overrightarrow{\mathrm{k}}=(2 \pi / \lambda) \overrightarrow{\mathrm{e}}_{k}$, a wave number in the direction of the unit propagation vector $\overrightarrow{\mathrm{e}}_{k}$.

Because in graphite purely two-dimensional layers are present, which have strong covalent internal forces and hence a large resistance against bending, the fourth-power term in Eq. (1c) may give rise to dispersion effects already for acoustic frequencies. For most layered structures, however, Komatsu's theory may not be used without some serious modifications, since the majority of these compounds do not display such an extreme crystallographic anisotropy as graphite. In fact, the constant $c_{33}$ may be of the same order of magnitude as the constants $c_{11}$ and $c_{12}$. On the other hand, the "layers" in the compounds under investigation are often built up from rather complicated clusters of atoms and hence the influence of $K$ may be relatively small at acoustic wavelengths.

In compounds with a large number of atoms per unit cell $(r)$, the acoustic modes of vibration only account for a rather small fraction of the total number of degrees of freedom. It has been suggested to describe only the acoustic-mode spectrum by a Debye-like approximation and to describe the optical-mode spectrum by $3 r-3$ suitably normalized $\delta$ functions located at some "average" optical-mode frequencies. However, apart from the fact that a large number of unknown parameters would be introduced, experimental evidence indicates that the optical-mode spectrum often appears to be rather "smeared out."14 Moreover, the assignment of the different branches of the dispersion relation of the lattice vibrations to "optical" and "acoustical" modes is unimportant for the calculation of the heat capacity. Therefore we will approximate the $3 r$ branches of the dispersion relation within the first Brillouin zone by three "pseudoelastic" branches, which are located within a modified Brillouin zone (MBZ).

The general problem will be treated as follows. First, we will describe the system by continuum elasticity theory, following a procedure somewhat analogous to the treatment of Bowman and Krumhansl. ${ }^{15}$ Next, the most dominating dispersion effects will be included by some suitably chosen MBZ boundaries. The dispersion at long wavelengths due to the intrinsic stiffness of layers or chains will be briefly considered in Sec. II. For the sake of clarity, the calculation below will be performed assuming a layered structure. The majority of the results, however, may be applied to chainlike compounds also, which will be pointed out in Sec. IV.

\section{A. Small- $k$ approximation}

The equations of motion of elastic waves in a continuum with uniaxial anisotropy are given by

$$
\begin{aligned}
\rho \frac{\partial^{2} u}{\partial t^{2}}= & c_{11} \frac{\partial^{2} u}{\partial x^{2}}+c_{66} \frac{\partial^{2} u}{\partial y^{2}}+\left(c_{12}+c_{66}\right) \frac{\partial^{2} v}{\partial x \partial y} \\
& +c_{44} \frac{\partial^{2} u}{\partial z^{2}}+\left(c_{13}+c_{44}\right) \frac{\partial^{2} w}{\partial x \partial z}, \\
\rho \frac{\partial^{2} v}{\partial t^{2}}= & c_{66} \frac{\partial^{2} v}{\partial x^{2}}+c_{11} \frac{\partial^{2} v}{\partial y^{2}}+\left(c_{12}+c_{66}\right) \frac{\partial^{2} u}{\partial x \partial y} \\
& +c_{44} \frac{\partial^{2} v}{\partial z^{2}}+\left(c_{13}+c_{44}\right) \frac{\partial^{2} w}{\partial y \partial z},
\end{aligned}
$$




$$
\begin{aligned}
\rho \frac{\partial^{2} w}{\partial t^{2}}= & c_{33} \frac{\partial^{2} w}{\partial z^{2}}+c_{44}\left(\frac{\partial^{2} w}{\partial x^{2}}+\frac{\partial^{2} w}{\partial y^{2}}\right) \\
& +\left(c_{13}+c_{44}\right)\left(\frac{\partial^{2} u}{\partial x \partial z}+\frac{\partial^{2} v}{\partial y \partial z}\right),
\end{aligned}
$$

where $\overrightarrow{\mathrm{x}}=(x, y, z)$, and $u, v$, and $w$ are the dis placements in the $x, y$, and $z$ direction, respectively.
Consider waves propagating in an infinite medium:

$$
\left[\begin{array}{l}
u \\
v \\
w
\end{array}\right]=\left[\begin{array}{c}
\xi \\
\eta \\
\zeta
\end{array}\right] e^{i(\overrightarrow{\mathrm{k}} \cdot \overrightarrow{\mathbf{x}}-\omega t)}
$$

Substitution into Eq. (2) yields the eigenvalue problem

$$
\left[\begin{array}{ccc}
c_{11} k_{x}^{2}+c_{66} k_{y}^{2}+c_{44} k_{z}^{2}-\rho \omega^{2} & \left(c_{12}+c_{66}\right) k_{x} k_{y} & \left(c_{13}+c_{44}\right) k_{x} k_{z} \\
\left(c_{12}+c_{66}\right) k_{x} k_{y} & c_{66} k_{x}^{2}+c_{11} k_{y}^{2}+c_{44} k_{z}^{2}-\rho \omega^{2} & \left(c_{13}+c_{44}\right) k_{y} k_{z} \\
\left(c_{13}+c_{44}\right) k_{x} k_{z} & \left(c_{13}+c_{44}\right) k_{y} k_{z} & c_{44}\left(k_{x}^{2}+k_{y}^{2}\right)+c_{33} k_{z}^{2}-\rho \omega^{2}
\end{array}\right]\left[\begin{array}{l}
\xi \\
\eta \\
\zeta
\end{array}\right]=0
$$

As a consequence of the hexagonal symmetry it is possible to separate out a solution corresponding to

$$
\rho \omega_{2}^{2}=c_{66}\left(k_{x}^{2}+k_{y}^{2}\right)+c_{44} k_{z}^{2} \text {. }
$$

This mode of vibration has a displacement in the $x y$ plane "transverse" with respect to $\overrightarrow{\mathrm{k}}$. The remaining eigenvalue problem is

$$
\left(\begin{array}{cc}
c_{11}\left(k_{x}^{2}+k_{y}^{2}\right)+c_{44} k_{z}^{2}-\rho \omega^{2} & \left(c_{13}+c_{44}\right) k_{z}\left(k_{x}^{2}+k_{y}^{2}\right)^{1 / 2} \\
\left(c_{13}+c_{44}\right) k_{z}\left(k_{x}^{2}+k_{y}^{2}\right)^{1 / 2} & c_{44}\left(k_{x}^{2}+k_{y}^{2}\right)+c_{33} k_{z}^{2}-\rho \omega^{2}
\end{array}\right)\left(\begin{array}{l}
\xi^{\prime} \\
\zeta
\end{array}\right)=0
$$

$\xi^{\prime}$ is located in the $x y$ plane at a direction perpendicular to the eigenvector that corresponds to Eq. (5).

If the off-diagonal elements in Eq. (6) are completely ignored, we obtain the approximate solutions

$$
\begin{aligned}
& \rho \omega_{1}^{2}=c_{11}\left(k_{x}^{2}+k_{y}^{2}\right)+c_{44} k_{z}^{2}, \\
& \rho \omega_{3}^{2}=c_{44}\left(k_{x}^{2}+k_{y}^{2}\right)+c_{33} k_{z}^{2} .
\end{aligned}
$$

The mode of vibration denoted by $\omega_{1}$ has a displacement in the plane "longitudinal" with respect to $\vec{k}$, while the mode denoted by $\omega_{3}$ has a displacement perpendicular to the $x y$ plane. The constant frequency contours of solutions (5) and (7) are ellipsoids in the $\overrightarrow{\mathrm{k}}$ space, which have rotational symmetry around the $k_{q}$ axis. For a large number of layered compounds the constant $c_{44}$ appears to be relatively small, and hence the curves presented in Fig. 1 may be fairly representative.

If the off-diagonal elements in Eq. (6) are taken into account, a rigorous calculation of the eigenvalues and eigenvectors shows that the modes of vibration given by Eq. (7) are coupled. The effect of such a coupling is shown in Fig. 2 for some representative values of the elastic constants. The drawn curves denote the constant-frequency contours in the diagonal approximation, while the dots represent the results obtained from a numerical calculation of the eigenvalues. The effect of the coupling is rather pronounced in the region where the drawn curves intersect, which corresponds to a cone in the $\overrightarrow{\mathrm{k}}$ space given by

$$
\left(k_{x}^{2}+k_{y}^{2}\right) / k_{z}^{2}=\left(c_{33}-c_{44}\right) /\left(c_{11}-c_{44}\right) .
$$

It can be seen from Fig. 2, however, that the correction is much smaller for most of the $\vec{k}$ space. Of course the direction of polarization is very sensitive to the coupling between the two different modes of vibration, but this has no consequence for the calculation of the heat capacity, and we feel that the diagonal approximation (7) provides a fair description of the dynamical behavior of the model.

\section{B. Dispersion effects}

As can be seen from Fig. 1 relatively small $\vec{k}$ vectors are associated with the "in-plane" modes propagating in the $x y$ plane. In the neighborhood of the $z$ direction, where the $\overrightarrow{\mathrm{k}}$ vector is relatively large, the elastic continuum approximation may very likely be incorrect, since the contours will reach the MBZ boundary for moderate values of $\omega$, which may give rise to rather drastic dispersion effects. In order to describe these effects we assert that for this mode of vibration, waves propagating in the layers may be considered as purely elastic, while dispersion effects near the $z$ direc- 


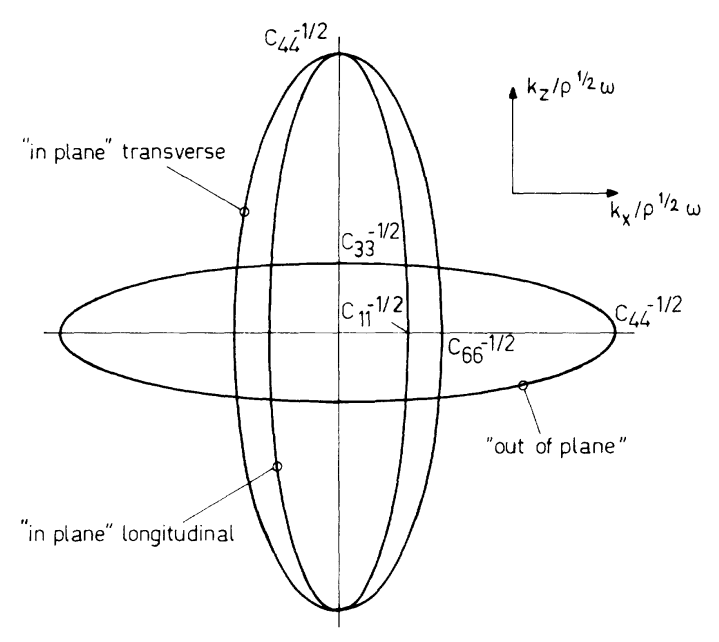

FIG. 1. Constant-frequency contours in the $\vec{k}$ space, which result from the diagonal approximation of the eigenvalue problem describing the equations of motion of elastic waves in a hexagonal layered structure. The meaning of the different vibrational modes is explained in the text.

tion may be taken into account by a MBZ parallel to the $x y$ plane located at $k_{z}= \pm \pi / 2 d$. "Truncation" at this boundary will occur if $k_{z}$ in Eq. (5) and (7a) is modified to $d^{-1} \sin \left(k_{z} d\right)$, while $k_{x}$ and $k_{y}$ remain unchanged. This modification yields the set of equations

$$
\begin{aligned}
& \rho \omega_{1}^{2}=c_{11}\left(k_{x}^{2}+k_{y}^{2}\right)+\left(c_{44} / d^{2}\right) \sin ^{2}\left(k_{z} d\right), \\
& \rho \omega_{2}^{2}=c_{66}\left(k_{x}^{2}+k_{y}^{2}\right)+\left(c_{44} / d^{2}\right) \sin ^{2}\left(k_{z} d\right) .
\end{aligned}
$$

Obviously these equations correspond exactly to the set of equations (1a) and (1b), which have been derived from a "thin-plate" model.

For the "out of plane" mode of vibration, however, the situation is quite different. The constant-frequency contour, given by Eq. (7b), appears to be more or less disc shaped, and hence dispersion effects will be important near the $x y$ plane rather than along the $z$ axis. These effects may be described by a cylinder shaped MBZ boundary located parallel to the $z$ axis at a radius $\pi / 2 d_{1}$, which transforms Eq. (7b) to

$$
\rho \omega_{3}^{2}=\left(c_{44} / d_{1}^{2}\right) \sin ^{2}\left[\left(k_{x}^{2}+k_{y}^{2}\right)^{1 / 2} d_{1}\right]+c_{33} k_{z}^{2} .
$$

\section{Bending stiffness}

Komatsu's treatment of the bond-bending problem of a monoatomic layer was based upon the

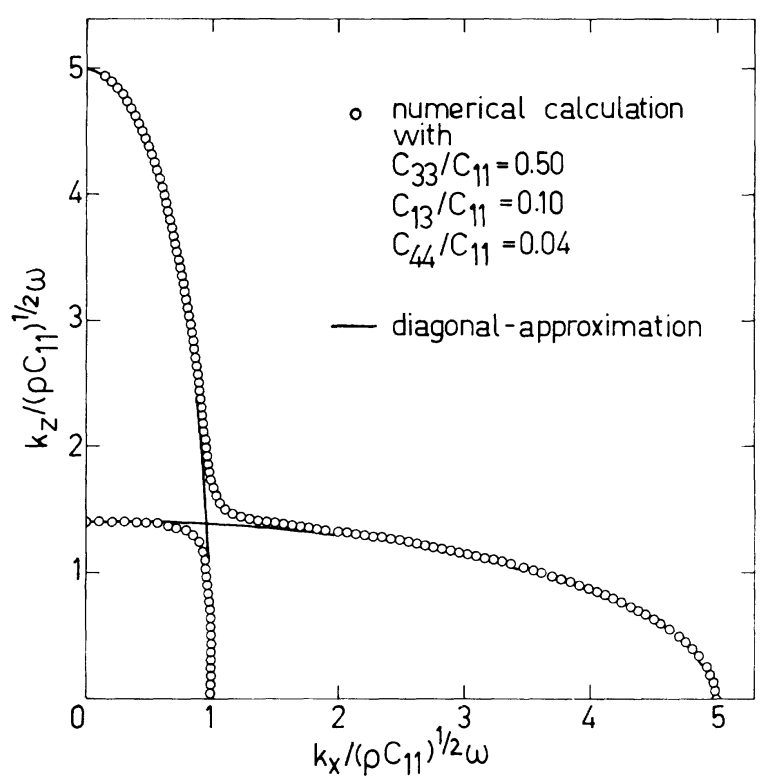

FIG. 2. An example of the effect of the introduction of the nondiagonal elements in the eigenvalue problem describing the elastic waves in a hexagonal medium.

assumption that the layer might be considered as a thin elastic plate. The validity of this assumption may be suitably examined by the atomistic model shown in Fig. 3, which represents a cross section perpendicular to the layers.

The different atoms-denoted by $n, m$-are arranged in a rectangular array, the spacing between adjacent atoms along the $x$ and $z$ axis being equal to $a$ and $d$, respectively. The array is assumed to resist variations of both the bond lengths and bond angles. Only nearest-neighbor interactions will be considered. In Fig. 4 the elementary de-
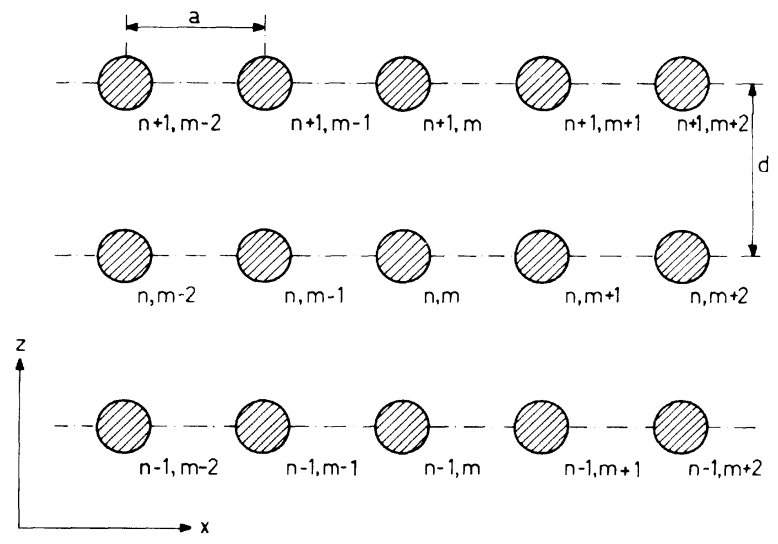

FIG. 3. A simple atomistic model used to describe the various interactions in an arbitrary plane perpendicular to the $x y$ layers. 
formations are given together with the corresponding increase in potential energy.

If both the kinetic energy $T$ and the potential energy $V$ are expressed in $u_{n, m}$ and $w_{n, m}$, which denote the atomic displacements along the $x$ and $z$ axis, respectively, the equations of motion may be found by applying Hamilton's principle

$$
\delta \int_{t_{1}}^{t_{2}}(T-V) d t=0
$$

The result is

$$
\begin{aligned}
M & \frac{\partial^{2} u_{n, m}}{\partial t^{2}}+\frac{C_{a}}{a^{2}}\left(2 u_{n, m}-u_{n, m+1}-u_{n, m-1}\right)+\frac{4 C_{\vartheta}}{d^{2}}\left(2 u_{n, m}-u_{n+1, m}-u_{n-1, m}\right) \\
+\left(\frac{a d}{l^{4}} C_{l}+\frac{1}{a d} C_{\vartheta}\right)\left(w_{n+1, m-1}+w_{n-1, m+1}-w_{n+1, m+1}-w_{n-1, m-1}\right) & +\frac{a^{2}}{l^{4}} C_{l}\left(4 u_{n, m}-u_{n+1, m+1}-u_{n-1, m-1}-u_{n+1, m-1}-u_{n-1, m+1}\right) \\
+ & +\frac{C_{\psi}}{d^{2}}\left(6 u_{n, m}-4 u_{n+1, m}-4 u_{n-1, m}+u_{n+2, m}+u_{n-2, m}\right)=0, \quad(1 \\
M \frac{\partial^{2} w_{n, m}}{\partial t^{2}}+\frac{C_{d}}{d^{2}}\left(2 w_{n, m}-w_{n+1, m}-w_{n-1, m}\right)+\frac{4 C_{\vartheta}}{a^{2}}\left(2 w_{n, m}-w_{n, m+1}-w_{n, m-1}\right) & \\
+\left(\frac{a d}{l^{4}} C_{l}+\frac{1}{a d} C_{\vartheta}\right)\left(u_{n+1, m-1}+u_{n-1, m+1}-u_{n+1, m+1}-u_{n-1, m-1}\right)+ & \frac{d^{2}}{l^{4}} C_{l}\left(4 w_{n, m}-w_{n+1, m+1}-w_{n-1, m-1}-w_{n+1, m-1}-w_{n-1, m+1}\right) \\
+ & +\frac{C_{\phi}}{a^{2}}\left(6 w_{n, m}-4 w_{n, m+1}-4 w_{n, m-1}+w_{n, m+2}+w_{n, m-2}\right)=0
\end{aligned}
$$

where $M$ denotes the atomic mass.

For long wavelengths the relative differences between the atomic displacements may be replaced by the corresponding derivatives to $x$ and $z$, and we obtain

$$
\begin{aligned}
M \frac{\partial^{2} u}{\partial t^{2}}= & \left(C_{a}+\frac{2 a^{4}}{l^{4}} C_{l}\right) \frac{\partial^{2} u}{\partial x^{2}}+\left(4 C_{\vartheta}+\frac{4 a^{2} d^{2}}{l^{4}} C_{l}\right) \frac{\partial^{2} w}{\partial x \partial z} \\
& +\left(4 C_{\vartheta}+\frac{2 a^{2} d^{2}}{l^{4}} C_{l}\right) \frac{\partial^{2} u}{\partial z^{2}}+C_{\Downarrow} d^{2} \frac{\partial^{4} u}{\partial z^{4}}, \\
M \frac{\partial^{2} w}{\partial t^{2}}= & \left(C_{d}+\frac{2 d^{4}}{l^{4}} C_{l}\right) \frac{\partial^{2} w}{\partial z^{2}}+\left(4 C_{\vartheta}+\frac{4 a^{2} d^{2}}{l^{4}} C_{l}\right) \frac{\partial^{2} u}{\partial x \partial z} \\
& +\left(4 C_{\vartheta}+\frac{2 a^{2} d^{2}}{l^{4}} C_{l}\right) \frac{\partial^{2} w}{\partial x^{2}}+C_{\phi} a^{2} \frac{\partial^{4} w}{\partial x^{4}} .
\end{aligned}
$$

As may be inferred from Eq. 2, continuum elasticity theory yields for the corresponding two-dimensional case

$$
\begin{aligned}
& \rho \frac{\partial^{2} u}{\partial t^{2}}=c_{11} \frac{\partial^{2} u}{\partial x^{2}}+\left(c_{13}+c_{44}\right) \frac{\partial^{2} w}{\partial x \partial z}+c_{44} \frac{\partial^{2} u}{\partial z^{2}}, \\
& \rho \frac{\partial^{2} w}{\partial t^{2}}=c_{33} \frac{\partial^{2} w}{\partial z^{2}}+\left(c_{13}+c_{44}\right) \frac{\partial^{2} u}{\partial x \partial z}+c_{44} \frac{\partial^{2} w}{\partial x^{2}} .
\end{aligned}
$$

It is obvious that

$$
\begin{aligned}
& c_{11}=\left(C_{a}+\frac{2 a^{4}}{l^{4}} C_{l}\right) \frac{\rho}{M}, \quad c_{33}=\left(C_{d}+\frac{2 d^{4}}{l^{4}} C_{l}\right) \frac{\rho}{M}, \\
& c_{13}=\frac{2 a^{2} d^{2} \rho}{l^{4} M} C_{l}, \quad c_{44}=\left(4 C_{\vartheta}+\frac{2 a^{2} d^{2}}{l^{4}} C_{l}\right) \frac{\rho}{M},
\end{aligned}
$$

and continuum elasticity theory gives a correct description of the long-wavelength limit of the vibrational spectrum. It appears, however, that the stiffness of $180^{\circ}$ bonds does not enter into the elastic constants. If the corresponding bending constants are extremely large, the influence of the fourth-power terms in (13) may be important already for acoustic frequencies, although such a drastic effect is likely to occur only for very anisotropic covalent substances like, for instance, graphite and boron nitride. For a description of the vibrational spectrum of these compounds, we may generalize Eq. (13) to three dimensions, and follow the procedure described in Sec. II B to obtain the dispersion relation

$$
\begin{aligned}
\rho \omega_{3}^{2}= & c_{44}\left(k_{x}^{2}+k_{y}^{2}\right)+\left(c_{33} / d^{2}\right) \sin ^{2}\left(k_{z} d\right) \\
& +C_{\phi}^{\prime} a^{2}\left(k_{x}^{2}+k_{y}^{2}\right)^{2} .
\end{aligned}
$$

The prime at $C_{\phi}$ is added to avoid confusion with the purely two-dimensional atomistic case. Equation (16) appears to be completely analogous to Eq. (1c) if we put $K^{2}=C_{\phi}^{\prime} a^{2}$. Equation (13a) will transform to Eq. (9a), because for a layered structure the effect of $C_{\psi}$ is negligible.

\section{CALCULATION OF THE HEAT CAPACITY}

A. General

In the diagonal approximation, the three modes of vibration are decoupled completely, and each mode will account for one third of the total number of degrees of freedom. In the calculation of 

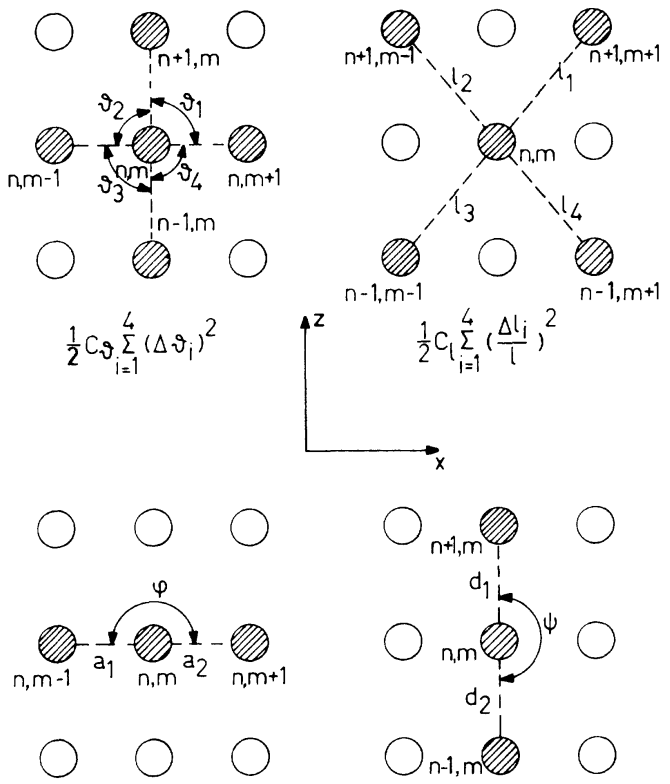

$$
\begin{gathered}
\frac{1}{2} C_{a}\left[\left(\frac{\Delta a_{1}}{a}\right)^{2}+\left(\frac{\Delta a_{2}}{a}\right)^{2}\right] \\
+\frac{1}{2} C_{\varphi}(\Delta \varphi)^{2}
\end{gathered}
$$$$
\begin{gathered}
\frac{1}{2} C_{d}\left[\left(\frac{\Delta d_{1}}{d}\right)^{2}+\left(\frac{\Delta d_{2}}{d}\right)^{2}\right] \\
+\frac{1}{2} C_{\psi}(\Delta \psi)^{2}
\end{gathered}
$$

FIG. 4. Some contributions to the increase of the potential energy, arising from variations of the bond angles and bond lengths in the atomistic model presented in Fig. 3.

the molar heat capacity, this number is assumed to amount to $3 r N_{\mathrm{AV}}$, where $N_{\mathrm{AV}}$ is Avogadro's number and $r$ is the number of vibrating units in a formula unit. The total specific heat may be obtained by a summation of the three properly nor malized contributions arising from the different modes of vibration.

The dispersion relations (9) and (10) are of two different types, given by

$$
\begin{gathered}
\omega^{2}=\alpha^{2}\left(k_{x}^{2}+k_{y}^{2}\right)+(\beta / d)^{2} \sin ^{2}\left(k_{z} d\right), \\
-\pi / 2 d \leqslant k_{z} \leqslant \pi / 2 d, \\
\omega^{2}=\left(\gamma / d_{1}\right)^{2} \sin ^{2}\left[\left(k_{x}^{2}+k_{y}^{2}\right)^{1 / 2} d_{1}\right]+\delta^{2} k_{z}^{2}, \\
0 \leqslant\left(k_{x}^{2}+k_{y}^{2}\right)^{1 / 2} \leqslant \pi / 2 d_{1} .
\end{gathered}
$$

In these equations $\alpha^{2}, \beta^{2}, \gamma^{2}$, and $\delta^{2}$ are combinations of the various elastic constants $c_{k 1} / \rho$. Since the sample size is normally very large compared to atomic dimensions, we will define a uniform density of states in the $\overrightarrow{\mathrm{k}}$ space, denoted by $\rho_{k}$. The different contributions to the heat capacity may then be evaluated rather straightforwardly. Let us consider Eq. (17) first.

By differentiating the number of vibrations with $\omega^{\prime}<\omega$ with respect to $\omega$, the frequency distribution function $g(\omega)$ can be found as

$$
\begin{aligned}
& g(\omega)=\rho_{k} \frac{4 \pi}{\alpha^{2} d} \omega \arcsin \left(\omega / \omega_{c}\right), \text { for } \omega \leqslant \omega_{c} \\
& g(\omega)=\rho_{k}\left(2 \pi^{2} / \alpha^{2} d\right) \omega, \text { for } \omega \geqslant \omega_{c} .
\end{aligned}
$$

In these expressions $\omega_{c}$ is written for $\beta / d$, the frequency at which "truncation" at the MBZ boundary occurs. The frequency distribution function is plotted in Fig. 5 , where $\omega_{m}$ denotes the "cutoff" frequency at which the normalization condition

$$
\rho_{k} \int_{0}^{\omega_{m}} g(\omega) d \omega=r N_{\mathrm{AV}}
$$

is satisfied. Substitution of Eq. (19) in (20) yields

$$
\rho_{k}\left(\pi^{2} / \alpha^{2} d\right)\left(\omega_{m}^{2}-\frac{1}{2} \omega_{c}^{2}\right)=r N_{\mathrm{AV}},
$$

from which it follows that

$g(\omega)=\frac{8 r N_{\mathrm{AV}}}{\pi\left(2 \omega_{m}^{2}-\omega_{c}^{2}\right)} \omega \arcsin \left(\omega / \omega_{c}\right)$, for $\omega \leqslant \omega_{c}$,

$g(\omega)=\frac{4 r N_{\mathrm{AV}}}{2 \omega_{m}^{2}-\omega_{c}^{2}} \omega$, for $\omega \geqslant \omega_{c}$.

As can be seen from these equations, the frequency distribution function $g(\omega)$ is determined completely by the magnitude of $\omega_{c}$ and $\omega_{m}$, which will be considered as independent parameters in the calculation of the heat capacity.

In general, the molar heat capacity $C(T)$ may be inferred from a normalized frequency distribution function $g(\omega)$ with the formula

$$
C(T)=k_{B} \int_{0}^{\omega m} \frac{g(\omega)(\hbar \omega / k T)^{2} e^{\hbar \omega / k T}}{\left(e^{\hbar \omega / k T}-1\right)^{2}} d \omega,
$$

where $k_{B}$ is the Boltzmann constant. If Eq. (22) is

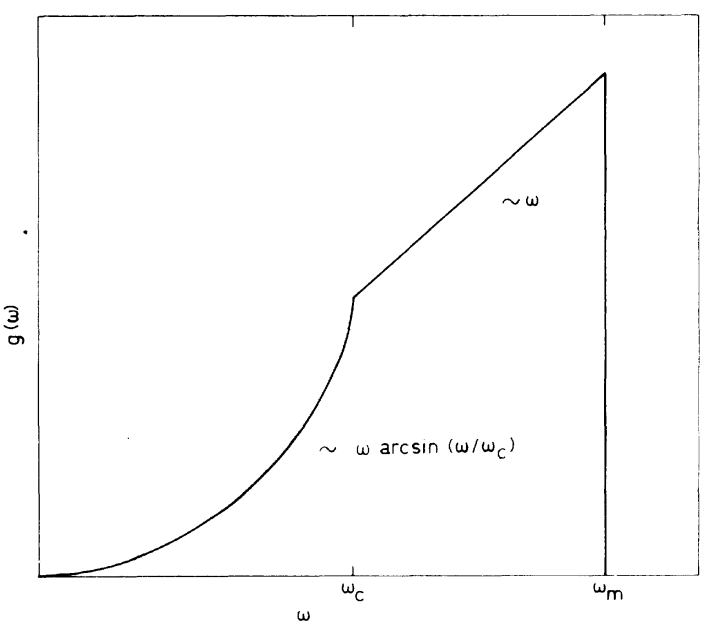

FIG. 5. The frequency distribution function $g(\omega)$ arising from a mode of vibration, for which dispersion effects near the $z$ axis are dominant. 
inserted in this expression, we obtain a contribution $F_{1}\left(\Theta_{m}, \Theta_{c}, T\right)$ to the heat capacity, given by

$$
\begin{aligned}
F_{1}\left(\Theta_{m}, \Theta_{c}, T\right) & \frac{r}{3\left(2 \Theta_{m}^{2}-\Theta_{c}^{2}\right)} \\
\times & {\left[\frac{24 R T^{2}}{\pi} \int_{0}^{\Theta_{c} / T} \frac{x^{3} e^{x}}{\left(e^{x}-1\right)^{2}} \arcsin \left(x T / \Theta_{c}\right) d x\right.} \\
& \left.+2 \Theta_{m}^{2} D_{2}\left(\Theta_{m} / T\right)-2 \Theta_{c}^{2} D_{2}\left(\Theta_{c} / T\right)\right]
\end{aligned}
$$

In this expression the usual substitutions

$x=\hbar \omega / k T, \quad \Theta_{c}=\hbar \omega_{c} / k, \quad \Theta_{m}=\hbar \omega_{m} / k$,

have been made, while $D_{2}(\Theta / T)$ denotes the twodimensional Debye function, defined in the Appendix.

Before we proceed with the evaluation of Eq. (18), we would like to make some remarks about the interpretation of the numerical values of $\omega_{c}$ and $\omega_{m}$. While $\omega_{c}$ has been defined as $\beta / d$ [cf. Eq. (19)], there is no direct relation between $\omega_{m}$ and the constant $\alpha$. Although it is not basically important for the calculation of the heat capacity, the value of $\omega_{m}$ may, to a certain extent, be associated with the magnitude of $\alpha$, which can be seen as follows. If we assume a cylinder shaped MBZ with height $\pi / d$ and radius $\pi / 2 d_{1}$, the volume of the MBZ will amount to $\pi^{4} / 4 d d_{1}^{2}$, and the corresponding density of states in the $\overrightarrow{\mathrm{k}}$ space, $\rho_{k}$, is found as

$$
\rho_{k}=4 d d_{1}^{2} r N_{\mathrm{AV}} / \pi^{4} .
$$

Of course, from a physical point of view, this assumption is not quite compatible with Eq. (17), since in this equation dispersion effects near the $x y$ plane are not taken into account, but in the present derivation of an approximate relation between $\omega_{m}$ and $\alpha$ the resulting error in the cutoff frequency of about a factor $\frac{1}{2} \pi$ is of no importance. Substitution of Eq. (26) in (21) yields the relation

$$
\omega_{m} \approx \frac{\pi}{2} \frac{\alpha}{d_{1}}\left(1+\frac{2 \beta^{2} d_{1}^{2}}{\pi^{2} \alpha^{2} d^{2}}\right)^{1 / 2} .
$$

Given the fact that $\alpha>\beta$ and that $d_{1}$ and $d$ are of the same order of magnitude, Eq. (27) shows that the value of $\omega_{m}$ may be used as an indication of the ratio $\alpha / d_{1}$.

Next we will consider Eq. (18). Following the same procedure as described above the frequency distribution function $g(\omega)$ may be found as

$$
g(\omega)=\frac{16 \pi \rho_{k}}{\alpha d_{1}^{2}} \int_{0}^{b} \zeta\left[1-\left(\omega_{c} / \omega\right)^{2} \sin ^{2} \zeta\right]^{-1 / 2} d \zeta,
$$

with $\omega_{c}=\gamma / d_{1}, \quad b=\arcsin \left(\omega / \omega_{c}\right)$ for $\omega \leqslant \omega_{c}$, and $b=\frac{1}{2} \pi$ for $\omega \geqslant \omega_{c}$. Since an analytical evaluation of the integral in this equation is not possible, the frequency distribution function has been computed numerically, and the result is plotted in Fig. 6 . The dashed line denotes the limit for $\omega \rightarrow \infty$, in which case Eq. (28) reduces to

$$
g(\omega)=\frac{16 \pi \rho_{k}}{\alpha d_{1}^{2}} \int_{0}^{\pi / 2} \zeta d \zeta=\frac{2 \pi^{3} \rho_{k}}{\alpha d_{1}^{2}} .
$$

In order to obtain a rather simple and manageable expression for the heat capacity involving only linear combinations of Debye functions, similar to Eq. (24), Eq. (28) will be used for $\omega<2 \omega_{c}$, and the limiting behavior (29) in the frequency range $\omega \geqslant 2 \omega_{c}$. It may be shown that this approximation produces only a small error in the magnitude of the heat capacity. For $\omega>\omega_{c}$ the number of vibrations $I(\omega)$ with $\omega^{\prime}<\omega$ is equal to

$$
I(\omega)=\frac{16 \pi \rho_{k} \omega_{c}}{\alpha d_{1}^{2}} \int_{0}^{\pi / 2} \zeta\left[\left(\omega / \omega_{c}\right)^{2}-\sin ^{2} \zeta\right]^{1 / 2} d \zeta,
$$

and hence the normalization condition (20) yields

$$
\begin{array}{r}
\frac{16 \pi \rho_{k}}{\alpha d_{1}^{2}}\left(\omega_{s} \int_{0}^{\pi / 2} \frac{\zeta}{2}\left(4-\sin ^{2} \zeta\right)^{1 / 2} d \zeta+\frac{\pi^{2}}{8}\left(\omega_{m}-\omega_{s}\right)\right) \\
=r N_{\mathrm{AV}},
\end{array}
$$

in which expression $\omega_{s}$ has been substituted for $2 \omega_{c}$. If we denote the integral in Eq. (31) by $I_{1}$, the frequency distribution function

$$
\begin{aligned}
g(\omega) & =\frac{8 r N_{\mathrm{AV}}}{\pi^{2} \omega_{m}-\left(\pi^{2}-8 I_{1}\right) \omega_{s}} \\
& \times \int_{0}^{b} \zeta\left[1-\left(\omega_{s} / 2 \omega\right)^{2} \sin ^{2} \zeta\right]^{-1 / 2} d \zeta,
\end{aligned}
$$

with

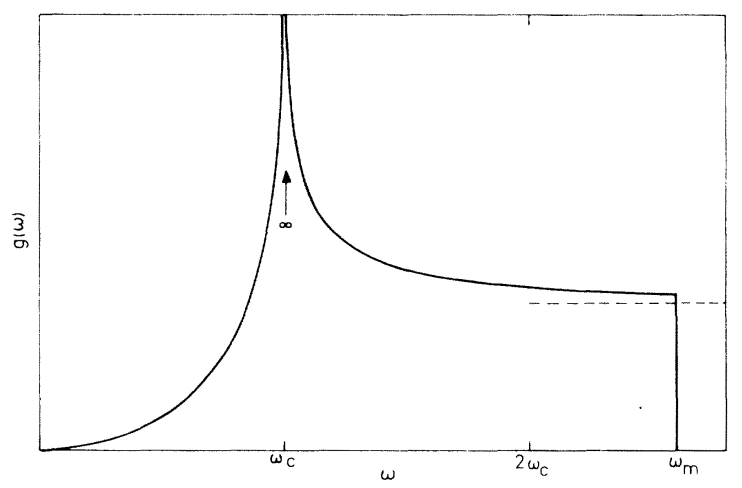

FIG. 6. The frequency distribution function $g(\omega)$ arising from a mode of vibration, for which dispersion effects near the $x y$ plane are dominant. The dashed line denotes the limiting behavior for $\omega \rightarrow \infty$. 


$$
\begin{aligned}
& b=\arcsin \left(2 \omega / \omega_{s}\right), \text { for } \omega \leqslant \frac{1}{2} \omega_{s} \\
& =\frac{1}{2} \pi \text { for } \frac{1}{2} \omega_{s} \leqslant \omega \leqslant \omega_{s}, \\
& g(\omega)=\frac{8 r N_{\mathrm{AV}}}{\pi^{2} \omega_{m}-\left(\pi^{2}-8 I_{1}\right) \omega_{s}} \frac{\pi^{2}}{8}, \text { for } \omega \geqslant \omega_{s}
\end{aligned}
$$

is obtained. Again, $g(\omega)$ is completely determined by the magnitude of $\omega_{s}$ and $\omega_{m}$. The same arguments that were applied in the evaluation of Eq. (17) may be used to show that the value of $\omega_{m}$ may now be associated with the ratio $\delta / d$.

If Eq. (32) is substituted in Eq. (23), we obtain a contribution $F_{2}\left(\Theta_{m}, \Theta_{s}, T\right)$ to the heat capacity given by

$$
\begin{aligned}
F_{2}\left(\Theta_{m}, \Theta_{s}, T\right)= & \frac{r}{3\left[\pi^{2} \Theta_{m}-\left(\pi^{2}-8 I_{1}\right) \Theta_{s}\right]} \\
& \times\left[8 I_{1} \Theta_{s} G_{2}\left(T / \Theta_{s}\right)+\pi^{2} \Theta_{m} D_{1}\left(\Theta_{m} / T\right)\right. \\
& \left.-\pi^{2} \Theta_{s} D_{1}\left(\Theta_{s} / T\right)\right],
\end{aligned}
$$

where $D_{1}(\Theta / T)$ denotes the one-dimensional Debye function, defined in the Appendix, where the function $G_{2}(T / \Theta)$ will be treated also.

\section{B. Layered structures}

As has been pointed out above, the lattice heat capacity $C_{L}(T)$ may be found by a summation of the three contributions arising from the different modes of vibration which may be written as

$C_{L}(T)=F_{1}\left(\Theta_{l}, \Theta_{c}, T\right)+F_{1}\left(\Theta_{t}, \Theta_{c}, T\right)+F_{2}\left(\Theta_{0}, \Theta_{s}, T\right)$.

In this expression $\Theta_{l}, \Theta_{t}$, and $\Theta_{0}$ are associated with the cutoff frequencies of the "longitudinal inplane," the "transverse in-plane," and the "out of plane" mode of vibration, respectively. The number of adjustable parameters in Eq. (34) amounts to 5 , but in order to keep this expression manageable in numerical fitting procedures, a further reduction of this number is generally imperative. Fortunately, such a reduction is often possible.

Firstly, the majority of the investigations on low-dimensional magnetic systems have been performed at rather low temperatures, in which case one "average" characteristic temperature may be used to describe the cutoff frequency of both the longitudinal and transverse in-plane modes of vibration. If the high-temperature region should be described more accurately, one might use the fact that the ratio $\Theta_{l} / \Theta_{t}$ is roughly equal to $v_{l} / v_{t}$, where $v_{l}$ and $v_{t}$ denote the propagation velocities of the longitudinal and transverse waves in the $x y$ plane, which are proportional to $\left(c_{11}\right)^{1 / 2}$ and $\left(c_{66}\right)^{1 / 2}$, respectively. Since for a wide variety of substances ${ }^{16}$ the ratio $c_{11} / c_{66}$ appears to range between 3 and 6 , the additional condition $\Theta_{l}=a \Theta_{t}$, with $1.5<a<2.5$, seems rather realistic.

Secondly, when the dimensions of the MBZ in the $x, y$, and $z$ direction are not too different, the problem will be simplified by the fact that "truncation" of the "cigar-shaped" contours in the $\vec{k}$ space occurs at the same frequency as the truncation of the "disc-shaped" contour, because for all contours the maximum $k$ value is proportional to $\left(c_{44}\right)^{-1 / 2}$. This yields the additional relation $\Theta_{s}$ $=2 \Theta_{c}$, which leaves only 3 independent parameters.

For extremely anisotropic substances, like graphite, boron nitride, and perhaps $\left(\mathrm{CH}_{3} \mathrm{NH}_{3}\right)_{2}$ $\mathrm{CdCl}_{4},{ }^{8}$ a bending modulus $\mathrm{K}$ should be included [cf. Eq. (16)]. We will, however, not consider this rather special case in the present treatment. For a calculation of the frequency spectrum for the out of plane mode of vibration and a discussion of the contribution to the heat capacity, the reader is referred to the literature. ${ }^{12,13}$

Finally, we would like to make some remarks about the application of Eq. (34) to the interpretation of experimental data. Both the integral on the right-hand side of Eq. (24) and the function $G_{2}(T / \Theta)$, which has been substituted in Eq. (33), cannot be evaluated analytically. With the aid of a high-speed computer they may be approximated with a very high degree of accuracy, but, especially when the functions $F_{1}$ and $F_{2}$ are used in numerical fitting procedures, the time involved with such a procedure is very large, since the various integrals have to be computed for each iteration and for all temperatures that correspond to the data points. Given the fact that the accuracy of most specific heat measurements is in the order of $1 \%$, we found it useful to deduce some rather simple expressions, which describe the various integrals with an accuracy of a few parts in $10^{4}$ for all values of $T / \Theta$. The derivation of these expressions will be given in the Appendix.

It should be noted that the functions $F_{1}$ and $F_{2}$ are only physically meaningful when the ratio $\Theta_{c} / \Theta_{m}$ and the ratio $\Theta_{s} / \Theta_{m}$ are small compared to unity. If the anisotropy for a particular mode of vibration accidentally appears to be very small, a description of the corresponding contribution with a suitably normalized three-dimensional Debye function is preferred.

\section{CHAINLIKE STRUCTURES}

In principle, the evaluation of the lattice dynamics of a chainlike structure is completely analogous to the problem treated in Secs. II and III, if the direction of the chains is chosen along the $z$ axis. Some modifications may arise from the fact that the relative magnitude of the elastic constants may be different from those of a layered structure. 
For a variety of anisotropic chainlike compounds, however, the shearing constant $c_{44}$ appears to be small compared to $c_{11}, c_{66}$, and $c_{33} \cdot{ }^{16}$ Therefore the contours presented in Fig. 1 will still be representative, except for the fact that the constant $c_{33}$, which now represents the compressional stiffness of the chains, will generally be larger than $c_{11}$ and $c_{66}$. As can easily be seen, this has no drastic consequence for the description of the dynamical behavior, given by continuum elasticity theory, and the expressions (9) and (10) are still valid. The dispersion relations denoted by $\omega_{1}, \omega_{2}$, and $\omega_{3}$ are now associated with the "out of chain longitudinal," the "out of chain transverse," and the "in chain" mode of vibration, respectively.

In general, the lattice heat capacity of chainlike substances may be described by Eq. (34) with 5 independent parameters. For these compounds, however, it is not obvious that both $\Theta_{l}$ and $\Theta_{t}$ are high compared to the temperature region in which the expression will be applied, and hence the additional condition $\Theta_{l}=a \Theta_{t}$ may produce some inac curacies in the description of the heat capacity. However, if the dimensions of the MBZ are not too different, the number of parameters can be reduced by the relation $\Theta_{s}=2 \Theta_{c}$, as has been pointed out in Sec. III.

To our knowledge, no heat capacity measurements have been reported on substances which are built up from very covalently bound purely onedimensional chains. Therefore the bending constant $C_{\psi}[\mathrm{cf} . \mathrm{Eq}$. (13)] has not been included in the present treatment.

\section{DISCUSSION}

In the preceding sections an approximation has been presented, which provides a rather general description of the lattice heat capacity of both layered and chainlike compounds. Since the inferred expressions involve only a few adjustable parameters, they are expected to be very useful in the interpretation of the heat capacity of lowdimensional magnetic substances. This is demonstrated by applying the theory to the description of the lattice contribution to the heat capacity of the series of isomorphic antiferromagnetic substances $\mathrm{CsMnC}_{3} \cdot 2 \mathrm{H}_{2} \mathrm{O}, \alpha \mathrm{RbMnCl}_{3} \cdot 2 \mathrm{H}_{2} \mathrm{O}$, and $\mathrm{CsMnBr}_{3} \cdot 2 \mathrm{H}_{2} \mathrm{O}$. These compounds may be considered as systems built up from layers of heavy ions, which are mainly held together by hydrogen bonds. ${ }^{17}$ Because in the paramagnetic region the magnetic properties of the different isomorphs are rather well established and the lattice contribution varies considerably, this series should offer a good indication of the applicability of the theory. Moreover, accurate experimental data were avail- able below $52 \mathrm{~K}$. We found that the lattice heat capacity for this series could be described very well between 9 and $52 \mathrm{~K}$ by Eq. (34) with 3 independent parameters. In fact, the errors were within the experimental uncertainty $(1 \%)$. The fitting procedure itself was found to be numerically stable, in contrast to a first attack on the problem, which was based upon a modification of the theory of Tarasov. ${ }^{7}$

Of course, a more direct check on the correctness of the description may be obtained by confronting the inferred expressions with the lowtemperature heat capacity of an anisotropic diamagnetic substance. Therefore we will briefly consider the heat capacity of $\left(\mathrm{CH}_{3}\right)_{4} \mathrm{NCdCl}_{3}$ (TMCC). Since detailed results of the measurements have been published elsewhere ${ }^{18}$ we will confine our selves to a discussion of the fitting procedure. In the interpretation of the heat capacity of TMCC one should note that this compound has been reported $^{19}$ to consist of chains of the form-Cd-C1 $1_{3}$ $\mathrm{Cd}-$, which are separated by $\mathrm{N}\left(\mathrm{CH}_{3}\right)_{4}$ complexes. Because at low temperatures the $\mathrm{CH}_{3}$ molecules will vibrate as a whole, the number of vibrating units in a formula unit was assumed to amount to 9. As a first attempt, the experimental data were described with the full Eq. (34), involving 5 independent parameters. The fitting procedure, however, revealed strong correlations between the parameters $\Theta_{l}, \Theta_{t}$, and $\Theta_{0}$, which is caused by the fact that the fit was performed at relatively low temperatures $(4<T<52 \mathrm{~K})$ where accurate experimental data were available. Because especially $\Theta_{0}$, reflecting the in-chain stiffness, showed a large standard deviation, we imposed the additional condition $\Theta_{0}=2 \Theta_{l}$. With the simplification $\Theta_{s}=2 \Theta_{c}$ the experimental data could be described with an accuracy better than $\sim 2 \%$. Only a slight improvement was obtained by considering $\Theta_{s}$ and $\Theta_{c}$ as independent variables, and hence the expression with three parameters was preferred. The result was

$\Theta_{l}=442 \pm 4 \mathrm{~K}, \Theta_{t}=154 \pm 1 \mathrm{~K}, \Theta_{c}=36.5 \pm 0.2 \mathrm{~K}$.

The same simplified form of Eq. (34) has been used to describe the lattice heat capacity of the isomorphic $\left(\mathrm{CH}_{3}\right)_{4} \mathrm{NMnCl}_{3}$ (TMMC). ${ }^{18}$ An excellent agreement with the experimental data was found, yielding $\Theta$ values which are about $8 \%$ higher than those for TMCC. This is consistent with the mass difference between the $\mathrm{Cd}^{++}$and the $\mathrm{Mn}^{++}$ion, if we assume that the binding forces are almost equal for both compounds.

Finally, we would like to make some concluding remarks. In the treatment of more complicated structures, one has to assume an "effective" number of vibrating units in a formula unit, denoted 
by $r$. In general, $r$ can be taken equal to the number of heavy atoms plus the number of molecular groups that are assumed to vibrate as a whole, at least within the temperature region that is of interest in the description of the heat capacity. The number of these groups-like $\mathrm{H}_{2} \mathrm{O}, \mathrm{CH}_{3}$, or $\mathrm{NH}_{3}$-may often be found from inspection of the crystallographic structure. Especially when the expressions are used in the description of the lowtemperature heat capacity, the quality of the fit appeared to be rather insensitive to small variations of the value of $r$. In the case of TMMC and TMCC, this value was assumed to be equal to 9 . Specific-heat measurements which have been performed on TMMC up till room temperature ${ }^{20}$ reveal that even at $300 \mathrm{~K}$ the total number of degrees of freedom hardly exceeds $27 N_{\mathrm{Av}}$, which strongly indicates that the conjectured value of $r$ is correct.

It has become common practice to describe experimental results with an apparent $\vartheta_{a}$ value, i.e., the value of $\Theta$ that should be inserted in a correctly normalized three-dimensional Debye function to predict the observed magnitude of the heat capacity at a given temperature $T$. The contribution $F_{2}$ can be described by an apparent $\Theta_{a}$ value defined by the equality

$$
\frac{1}{3} D_{3}\left(\Theta_{a} / T\right)=F_{2}\left(\Theta_{m}, \Theta_{s}, T\right) .
$$

The result is presented in Fig. 7, where the ratio $\Theta_{a} / \Theta_{m}$ has been normalized to unity at $T=0$. A similar behavior is found for the contribution $F_{1}$. It should be noted that even for small values of the anisotropy our theory predicts a minimum of $\Theta_{a}$ in the temperature region $0.02<T / \Theta_{m}<0.2$, which has also been observed in a considerable number of experimental investigations. ${ }^{21}$ Hence

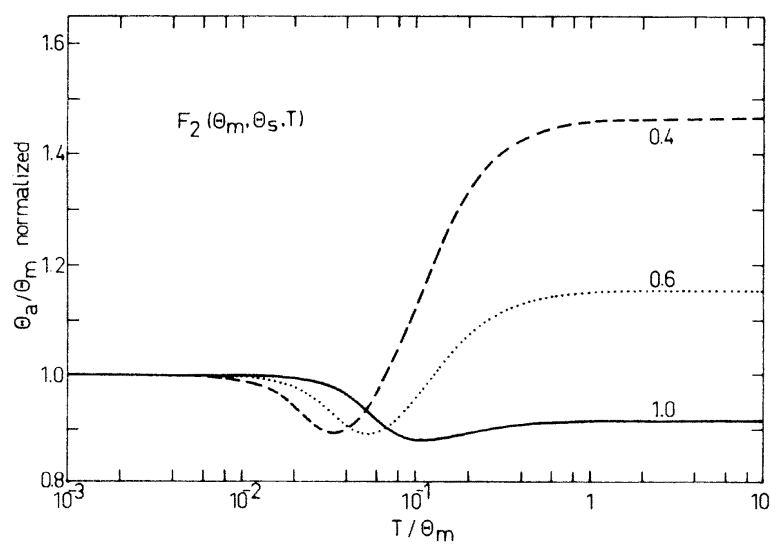

FIG. 7. Description of the contribution to the specific heat, arising from a mode of vibration for which dispersion effects near the $x y$ plane are dominant, with an apparent $\Theta_{a}$ value. The different curves are characterized by the ratio $\Theta_{s} / \Theta_{m}$. it appears that the most essential shortcoming of the purely elastic Debye model in the description of the low-temperature heat capacity may already be removed by the inclusion of only the most dominant dispersion effects. The limiting $T^{3}$ dependence of the heat capacity appears to occur only at temperatures very low compared to the region in which the "Debye $T^{3}$ law" mathematically holds. If the compound under investigation has a fair amount of anisotropy, conventional techniques to separate the electronic or magnetic contribution from the total specific heat, such as a $C / T$ versus $T^{2}$ or a $C T^{2}$ versus $T^{5}$ plot, respectively, should only be applied with great care, since they are based upon a purely $T^{3}$ dependence of the lattice heat capacity.

\section{ACKNOWLEDGMENTS}

The authors wish to thank J. P. A. M. Hijmans for some valuable suggestions and Professor F. van der Maesen for critical reading of the manuscript. We also like to acknowledge some stimulating discussions with Professor L. J. F. Broer.

\section{APPENDIX}

The $n$-dimensional Debye function used in this paper is defined as

$$
D_{n}(y)=\frac{3 n R}{y} \int_{0}^{y} \frac{x^{n+1} e^{x}}{\left(e^{x}-1\right)^{2}} d x,
$$

where $R$ denotes the molar gas constant.

The integral that appears at the right-hand side of Eq. (24) can be evaluated as follows. Let us define a function $G_{1}\left(T / \Theta_{c}\right)$ as

$G_{1}\left(\frac{T}{\Theta_{c}}\right)=\frac{24 R T^{2}}{\pi \Theta_{c}^{2}} \int_{0}^{\Theta_{c} / T} \frac{x^{3} e^{x}}{\left(e^{x}-1\right)^{2}} \arcsin \left(\frac{x T}{\Theta_{c}}\right) d x$.

The low-temperature behavior of $G_{1}\left(T / \Theta_{c}\right)$ can be found by substituting $\arcsin \left(x T / \boldsymbol{\Theta}_{c}\right)=x T / \boldsymbol{\Theta}_{c}$, since the integrand goes exponentially to zero for large values

TABLE I. Coefficients $B_{1, i}$ of the polynomial series $P_{1}\left(T / \Theta_{c}\right)$ that relates the function $G_{1}\left(T / \Theta_{c}\right)$ to a threedimensional Debye function.

\begin{tabular}{cc}
\hline \hline$T / \Theta_{c} \leq 0.1$ & $T / \Theta_{c} \geq 0.1$ \\
\hline$B_{1,0}=+2.43502 \times 10^{2}$ & $B_{1,0}=-5.63143$ \\
$B_{1,1}=+7.11922 \times 10^{2}$ & $B_{1,1}=-1.94211$ \\
$B_{1,2}=+8.66012 \times 10^{2}$ & $B_{1,2}=-5.32241 \times 10^{-2}$ \\
$B_{1,3}=+5.87204 \times 10^{2}$ & $B_{1,3}=+3.39971 \times 10^{-2}$ \\
$B_{1,4}=+2.46622 \times 10^{2}$ & $B_{1,4}=-1.88309 \times 10^{-2}$ \\
$B_{1,5}=+6.70161 \times 10$ & $B_{1,5}=+7.53021 \times 10^{-3}$ \\
$B_{1,6}=+1.18407 \times 10$ & $B_{1,6}=-1.48747 \times 10^{-3}$ \\
$B_{1,7}=+1.31640$ & $B_{1,7}=-5.64074 \times 10^{-8}$ \\
$B_{1,8}=+8.37847 \times 10^{-2}$ & $B_{1,8}=+4.33673 \times 10^{-5}$ \\
$B_{1,9}=+2.33086 \times 10^{-3}$ & $B_{1,9}=-4.44569 \times 10^{-6}$ \\
\hline \hline
\end{tabular}


TABLE II. Coefficients $B_{2, i}$ of the polynomial series $P_{2}\left(T / \Theta_{s}\right)$ that relates the function $G_{2}\left(T / \Theta_{s}\right)$ to a three-dimensional Debye function.

\begin{tabular}{cll}
\hline \hline$T / \Theta_{S} \leq 0.03$ & \multicolumn{1}{c}{$0.03 \leq T / \Theta_{s} \leq 0.40$} & \multicolumn{1}{c}{$0.40 \leq T / \Theta_{s}$} \\
\hline$B_{2,0}=-2.49859 \times 10^{4}$ & $B_{2,0}=-3.00059$ & $B_{2,0}=-4.94117$ \\
$B_{2,1}=-7.32324 \times 10^{4}$ & $B_{2,1}=+8.17766$ & $B_{2,1}=-2.00101$ \\
$B_{2,2}=-9.28342 \times 10^{4}$ & $B_{2,2}=+2.37706 \times 10$ & $B_{2,2}=+7.11166 \times 10^{-4}$ \\
$B_{2,3}=-6.70697 \times 10^{4}$ & $B_{2,3}=+3.24892 \times 10$ & $B_{2,3}=+4.36995 \times 10^{-4}$ \\
$B_{2,4}=-3.05181 \times 10^{4}$ & $B_{2,4}=+2.86322 \times 10$ & $B_{2,4}=-1.73992 \times 10^{-3}$ \\
$B_{2,5}=-9.08819 \times 10^{3}$ & $B_{2,5}=+1.68368 \times 10$ & $B_{2,5}=+1.71293 \times 10^{-3}$ \\
$B_{2,6}=-1.77404 \times 10^{3}$ & $B_{2,6}=+6.57270$ & $B_{2,6}=-8.19824 \times 10^{-4}$ \\
$B_{2,7}=-2.19161 \times 10^{2}$ & $B_{2,7}=+1.63229$ & $B_{2,7}=+2.09085 \times 10^{-4}$ \\
$B_{2,8}=-1.55637 \times 10^{-1}$ & $B_{2,8}=+2.31085 \times 10^{-1}$ & $B_{2,8}=-2.72743 \times 10^{-5}$ \\
$B_{2,9}=-4.84491 \times 10^{-1}$ & $B_{2,9}=+1.40476 \times 10^{-2}$ & $B_{2,9}=+1.43144 \times 10^{-6}$ \\
\hline \hline
\end{tabular}

of $x$. The result is

$$
G_{1}\left(T / \Theta_{c}\right)=(8 / 3 \pi) D_{3}\left(\Theta_{c} / T\right)
$$

At low temperatures, the function $G_{1}$ may also be described by the equation $G_{1}\left(T / \Theta_{c}\right)=D_{3}(\Theta / T)$, if we put

$$
\Theta=(3 \pi / 8)^{1 / 3} \Theta_{c} .
$$

At higher temperatures, the relative differences between the function $G_{1}\left(T / \Theta_{c}\right)$ and the three-dimensional Debye function $D_{3}\left[(3 \pi / 8)^{1 / 3} \Theta_{c} / T\right]$ may now be approximated with a function $P_{1}\left(T / \Theta_{c}\right)$, for example, a polynomial series in $T / \Theta_{c}$. The approximation

$$
G_{1}\left(T / \Theta_{c}\right)=D_{3}\left[\left(\frac{3}{8} \pi\right)^{1 / 3} \Theta_{c} / T\right]\left[1-P_{1}\left(T / \Theta_{c}\right)\right]
$$

was found to have a relative accuracy better than $2 \times 10^{-4}$ for $0<T / \Theta_{c}<\infty$ with the polynomial series

$$
P_{1}\left(T / \Theta_{c}\right)=\exp \left(\sum_{i=0}^{9} B_{1, i}\left[\ln \left(T / \Theta_{c}\right)\right]^{i}\right) .
$$

The coefficients $B_{1, i}$, obtained by a least-squares fit of approximation (40), are listed in Table I.

The function $G_{2}(T / \Theta)$, substituted in Eq. (33), is equal to the heat capacity that is obtained if the frequency distribution function

$$
g(\omega)=\frac{3}{\omega_{s} I_{1}} \int_{0}^{b} \zeta\left[1-\left(\omega_{s} / 2 \omega\right)^{2} \sin ^{2} \zeta\right]^{-1 / 2} d \zeta,
$$

with $b=\arcsin \left(2 \omega / \omega_{s}\right)$ for $\omega \leqslant \frac{1}{2} \omega_{s}$ and $b=\frac{1}{2} \pi$ for $\omega \geqslant \frac{1}{2} \omega_{s}$, is substituted into Eq. (23) with $\omega_{m}=\omega_{s}$.
For $\omega \ll \omega_{s}$, Eq. (42) reduces to

$$
\begin{aligned}
g(\omega) & =\frac{3}{\omega_{s} I_{1}} \int_{0}^{2 \omega / \omega} s\left[1-\left(\omega_{s} / 2 \omega\right)^{2} \zeta^{2}\right]^{-1 / 2} d \zeta \\
& =\frac{12 \omega^{2}}{I_{1} \omega_{s}^{3}} .
\end{aligned}
$$

The iimiting low-temperature behavior of the heat capacity may now be found as

$$
G_{2}\left(T / \Theta_{s}\right)=4 /\left(3 I_{1}\right) D_{3}\left(\Theta_{s} / T\right),
$$

or alternatively,

$$
G_{2}\left(T / \Theta_{s}\right)=D_{3}\left[\left(3 I_{1} / 4\right)^{1 / 3} \Theta_{s} / T\right] .
$$

Following the same procedure as outlined above the function $G_{2}\left(T / \Theta_{s}\right)$ can be described with a relative accuracy better than $5 \times 10^{-4}$ for all temperatures by the approximation

$$
G_{2}\left(T / \Theta_{s}\right)=D_{3}\left[\left(3 I_{1} / 4\right)^{1 / 3} \Theta_{s} / T\right]\left[1-P_{2}\left(T / \Theta_{s}\right)\right] .
$$

The magnitude of $I_{1}$ has been numerically evaluated as $I_{1}=1.1190677$, while the constants $B_{2, i}$ in the polynomial series $P_{2}\left(T / \Theta_{s}\right)$, having the same functional form as $P_{1}\left(T / \Theta_{c}\right)$, are listed in Table II.

Given the fact that for the usual Debye functions various series expansions are available,$^{22}$ the results given by the relations (40) and (46) are very suitable in numerical fitting procedures, since the derivatives to the different parameters may be inferred very easily.
${ }^{1}$ C. Domb and A. R. Miedema, Prog. Low Temp. Phys. 4, 296 (1964).

${ }^{2}$ I. F. Schegolev, Phys. Status Solidi A 12, 9 (1972).

${ }^{3}$ L. J. de Jongh and A. R. Miedema, Adv. Phys. 23, 1 (1974).

${ }^{4}$ K. Komatsu and T. Nagamiya, J. Phys. Soc. Jpn. $\underline{6}$, 438 (1951).

${ }^{5}$ J. A. Hofmann, A. Paskin, K. J. Tauer, and R. J. Weiss,
J. Phys. Chem. Solids 1, 45 (1956).

${ }^{6} \mathrm{~V} . \mathrm{V}$. Tarasov, New problems in the Physics of Glass (Oldbourne, London, 1963).

${ }^{7}$ K. Kopinga, T. de Neef, and W. J. M. de Jonge, Phys. Rev. B 11, 2364 (1975).

${ }^{8}$ P. Bloembergen and A. R. Miedema, Physica (Utr.) 75 , 205 (1974).

${ }^{9}$ L. D. Jennings and W. N. Hansen, Phys. Rev. 139, 


\author{
A1694 (1965). \\ ${ }^{10}$ K. Blacklock, H. F. Linebarger, H. W. White, K. H. \\ Lee, and S. L. Holt, J. Chem. Phys. 61, 5279 (1975). \\ ${ }^{11}$ References can be found, for instance, in K. K. Mani \\ and R. Ramani, Phys. Status Solidi 61, 659 (1974); \\ G. F. Newell, J. Chem. Phys. 27, 240 (1957). \\ ${ }^{12}$ K. Komatsu, J. Phys. Soc. Jpn. 10, 346 (1955). \\ ${ }^{13}$ K. Komatsu, J. Phys. Chem. Solids 6 , 380 (1958). \\ ${ }^{14} \mathrm{~J}$. H. M. Stoelinga and P. Wyder, J. Chem. Phys. 61 , \\ 478 (1974). \\ ${ }^{15}$ J. C. Bowman and J. A. Krumhansl, J. Phys. Chem. \\ Solids $\underline{6}, 367$ (1958). \\ ${ }^{16}$ R. Bechmann, R. F. S. Hearman, and S. K. Kurtz, in \\ Landolt-Bornstein, Numerical Data and Functional \\ Relationships in Science and Technology, Group III, \\ Vol. 1, 2 (Springer-Verlag, Berlin, 1966).
}

${ }^{17}$ S. J. Jensen, P. Andersen, and S. E. Rasmussen, Acta Chem. Scand. 16, 1890 (1962).

${ }^{18}$ W. J. M. de Jonge, C. H. W. Swüste, K. Kopinga, and K. Takeda, Phys. Rev. B 12, 5858 (1975).

${ }^{19}$ P. S. Peercy, B. Morosin, and G. A. Samara, Phys. Rev. B 8, 3378 (1973).

${ }^{20}$ R. E. Dietz, L. R. Walker, F. S. L. Hsu, W. H. Haemmerle, B. Vis, C. K. Chau, and H. Weinstock, Solid State Commun. 15, 1185 (1974).

${ }^{21}$ A. A. Maradudin, E. W. Montroll, G. H. Weiss, and I. P. Ipatova, Theory of the Lattice Dynamics in the Harmonic Approximation (Academic, New York, 1971). ${ }^{22}$ M. Abramowitz and I. A. Stegun, Handbook of Mathematical Functions [Natl. Bur. Stand. (U.S.) No. 55, 1964]. 